\title{
Target-controlled infusion of propofol and remifentanil combined with dexmedetomidine reduces functional endoscopic sinus surgery bleeding
}

\author{
DENG-FENG DING $^{1 *}$, LI-FANG WU ${ }^{2 *}$, PING WANG $^{1}$, YUAN-XU JIANG $^{1}$, \\ YAO-WEN LUO ${ }^{1}$, ZHONG-LIANG DAI $^{1}$, XUE-PING ZHANG ${ }^{1}$ and YA-LI LI ${ }^{1}$ \\ ${ }^{1}$ Department of Anesthesiology, Shenzhen People's Hospital, Jinan University, Shenzhen, \\ Guangdong 518020; ${ }^{2}$ Department of Anesthesiology, The First Affiliated Hospital of Inner \\ Mongolia Medical University, Hohhot, Inner Mongolia 010000, P.R. China
}

Received May 7, 2016; Accepted April 7, 2017

DOI: $10.3892 /$ etm.2017.5075

\begin{abstract}
The aim of the present study was to investigate the effects of target-controlled infusion (TCI) of propofol and remifentanil combined with dexmedetomidine on functional endoscopic sinus surgery (FESS) bleeding and surgical field. 62 patients scheduled to undergo FESS were randomly divided into experimental group (intravenous $0.5 \mu \mathrm{g} \mathrm{kg}^{-1} \mathrm{~h}^{-1}$ dexmedetomidine after $0.5 \mu \mathrm{g} \mathrm{kg}^{-1}$ bolus within $15 \mathrm{~min}$ until the end of surgery) or control group (intravenous saline administration at the same dose). All patients underwent endotracheal intubation under general anesthesia with TCI of propofol and remifentanil for anesthesia induction and maintenance. During anesthesia, arterial pressure (MAP), heart rate (HR), intraoperative propofol and remifentanil dosage and intraoperative blood loss were recorded. Surgeons rated their satisfaction with the surgical field using the Numeric Rating Scale (NRS). Following surgery, visual analog scale (VAS) was assessed. During tracheal intubation and extubation, HR and MAP in the experimental group were significantly lower compared with the control group $(\mathrm{P}<0.05)$; HR was also significantly lower compared with the control group throughout surgery $(\mathrm{P}<0.05)$. The mean infusion rate of propofol and remifentanil was significantly lower in the experimental group compared with the control group $(\mathrm{P}=0.001$ and $\mathrm{P}=0.045$, respectively). Blood loss in the experimental group was significantly lower
\end{abstract}

Correspondence to: Dr Xue-Ping Zhang or Professor Ya-Li Li, Department of Anesthesiology, Shenzhen People's Hospital, Jinan University, 1017 North Dongmen Road, Shenzhen, Guangdong 518020, P.R. China

E-mail: xuepzhang@sina.com

E-mail: yalieledr@sina.com

*Contributed equally

Key words: propofol, remifentanil, dexmedetomidine, functional endoscopic sinus surgery compared with the control group $(\mathrm{P}=0.007)$. NRS and VAS scores in the experimental group were significantly improved compared with control group $(\mathrm{P}<0.01)$. In conclusion, TCI of propofol and remifentanil for FESS combined with dexmedetomidine reduced intraoperative bleeding and improved the quality of surgical field compared with the same procedure without dexmedetomidine. Dexmedetomidine also reduced the increase in MAP and HR during tracheal intubation and extubation, and improved postoperative analgesia quality.

\section{Introduction}

Functional endoscopic sinus surgery (FESS) is a minimally invasive technique used to restore nasal cavity ventilation and sinus function (1). Bleeding is an important challenge for clinicians to overcome in FESS. Due to the enriched blood vessels in the nasal cavity and hemostasis difficulty, bleeding not only hinders the surgical field of FESS, but also prolongs the duration of surgery time and increases the risk of complications (2).

Various methods are used to reduce bleeding during FESS surgery, but controlled hypotension remains an important factor (3). In the past, vasoactive drugs were used for the control of blood pressure; however, these agents are prone to causing hemodynamic instability and, in severe cases, even organ ischemia leading to significant organ dysfunction (4). Currently, anesthesia techniques and drugs are more commonly used for controlling blood pressure and heart rate (HR), in order to reduce FESS bleeding (5). Previous studies have reported that the use of propofol and remifentanil for total intravenous anesthesia has a notable advantage in reducing bleeding compared with inhalation anesthesia (6-8), which has made propofol and remifentanil for total intravenous anesthesia the preferred method for FESS surgery anesthesia. However, there may still be room for improvement in reducing intraoperative bleeding and improving the surgical field. At present, there is little research in this area.

Dexmedetomidine is a highly selective $\alpha 2$ adrenoceptor agonist which inhibits activity of the sympathetic nervous system, reduces the release of catecholamines, lowers blood pressure and slows the HR (9). Previous studies have indicated 
that dexmedetomidine, like remifentanil, is effective in controlling blood pressure and maintaining hemodynamic stability, so as to achieve the same surgical field as remifentanil $(10,11)$. However, it is not yet known what the effects would be if these two drugs were used in combination.

The primary objective of this prospective, randomized, controlled study was to evaluate the effect of dexmedetomidine combined with target-controlled infusion (TCI) of propofol and remifentanil on FESS bleeding and the surgical field.

\section{Materials and methods}

Patients and characteristics. The present study was approved by the Ethics Committee of Shenzhen People's Hospital (Shenzhen, China). Written informed consent was obtained from all participants. A total of 62 patients undergoing elective FESS surgery, American Society of Anesthesiologists (ASA) class I-II, aged 19-53 years old, participated in the present study between September 2013 and January 2016. Exclusion criteria were as follows: body mass index $\geq 25$, HR $<50$ times per min, heart block, hypertension, coronary heart disease, diabetes, history of asthma, chronic obstructive emphysema, treatments with antibiotics or anticoagulants, coagulation abnormalities, failure of cooperation because of mental disorders, and use of sedative and analgesic drugs within $48 \mathrm{~h}$ before surgery. An anesthesiologist, who participated in the design of the grouping but not in anesthesia and data collection, assigned dexmedetomidine. A different anesthesiologist performed clinical anesthesia and data collection. Surgeons and patients were blinded to the groupings.

Patient grouping. Patients were randomly divided into an experimental group and a control group with 31 cases in each group. In the experimental group, the procedure was as following: Before the induction of anesthesia, infusion of $0.5 \mu \mathrm{g} \mathrm{kg}^{-1}$ dexmedetomidine was performed within $15 \mathrm{~min}$, followed by $0.5 \mu \mathrm{g} \mathrm{kg}^{-1} \mathrm{~h}^{-1}$ dexmedetomidine via intravenous pump continuous injection until the end of the surgery. The control group were administered with an intravenous pump injection normal saline equivalent to the volume of dexmedetomidine administered in the experimental group. All patients entered the operating room without pre-medication and having fasted for 8-12 h. An anesthesia monitor was used (Compact Anesthesia Monitor; GE Healthcare, Chicago, IL, USA) and routine electrocardiogram monitoring was carried out. Saturation of peripheral oxygen $\left(\mathrm{SpO}_{2}\right)$ and end tidal carbon dioxide $\left(\mathrm{PetCO}_{2}\right)$ were monitored. Depth of anesthesia was measured using a bispectral index (BIS) system (BIS Vista Monitoring System, Aspect Medical Systems; Covidien, Dublin, Ireland). All patients were subjected to tracheal intubation and general anaesthesia with TCI of propofol and remifentanil to maintain the effect-site concentration. Oxygen ventilation of $51 \cdot \mathrm{min}^{-1}$, was administered $5 \mathrm{~min}$ before using commercial TCI system (Base Observational, Fresenius Kabi AG, Bad Homburg, Germany). TCI consisted of $4 \mu \mathrm{g} \mathrm{ml}^{-1}$ propofol (Schnider model) and $3 \mathrm{ng} \mathrm{ml}^{-1}$ remifentanil (Minto model) for anesthesia induction of (12). Tracheal intubation was performed $120 \mathrm{sec}$ after administration of intravenous injection of $0.2 \mathrm{mg} \mathrm{kg}^{-1}$ cisatracurium ammonium and mask-assisted ventilation following TCI (endotracheal tube diameter, $7.0 \mathrm{~mm}$ for females and $7.5 \mathrm{~mm}$ for males). Subsequently, endotracheal tube was connected with the anesthesia machine (Primus; Draeger Medical Ag \& Co. KGaA, Luebeck, Germany) to control the breathing, and respiratory parameters were adjusted to the following: Tidal volume, $6-10 \mathrm{ml} \mathrm{kg}^{-1}$; respiratory rate, 12-16 times/min; inspiration/expiration ratio, 1:2; $\mathrm{PetCO}_{2}$ maintained at $35-45 \mathrm{mmHg}$; and airway pressure maintained at $10-20 \mathrm{mmH}_{2} \mathrm{O}$. TCI of $2-8 \mu \mathrm{g} \mathrm{ml}^{-1}$ propofol and 2-6 ng ml-1 remifentanil was applied to maintain anesthesia. Through adjusting the dosage of the propofol and remifentanil, BIS value was maintained at 45-55 and mean arterial pressure (MAP) was maintained at $70-80 \mathrm{mmHg}$. Intravenous injection of $0.25 \mathrm{mg}$ atropine was administered if HR was $<50$ times/min, which was considered as bradycardia.

Surgery. All patients were in the supine position for surgery. Prior to the start of surgery, four pieces of tampon, soaked in adrenaline and lidocaine mixture solution $(0.1 \%$ epinephrine: $2 \%$ lidocaine, 1:1) were used to shrink the blood vessels in the nasal mucosa. All surgical procedures were performed by the same surgeon using standard procedures.

At the end of surgery, propofol and remifentanil were replaced with intravenous injection of $40 \mathrm{mg}$ parecoxib sodium for postoperative analgesia. Patients were moved into Postanesthesia Care Unit (PACU), where another anesthesiologist, responsible for recovery from anesthesia, finished extubation after patients were awake. $1 \mu \mathrm{g} \mathrm{kg}^{-1}$ fentanyl was administered if visual analog scale (VAS) score was $>5$ in the PACU.

Intra-operative variables and recovery profiles for patients. In the operating room, MAP and HR were recorded at baseline (T0), before anesthesia induction (T1), before tracheal intubation (T2), at the moment of tracheal intubation (T3), before the start of surgery (T4), 15 min after the start of surgery (T5), at the end of surgery (T6) and at the moment of extubation (T7).

The following parameters were recorded: Duration of surgery, duration of anesthesia (from anesthesia induction to anesthetic drug withdrawal), consumption of propofol and remifentanil, incidence of bradycardia and blood loss. Following completion of the surgery, surgeons evaluated the surgical field using the Numeric Rating Scale (NRS), from 0 (worst) to 10 (best) (10). Extubation time (from anesthetic drug withdrawal to extubation and respiratory rate after extubation were recorded. The Observer's Assessment of Alertness/Sedation (OAA/S) scale (13) was used to assess sedation $5 \mathrm{~min}$ after extubation and pain severity was recorded using VAS (11) before the patient left the recovery room. The use of fentanyl was observed in recovery room. Hypoxemia after extubation $\left(\mathrm{SpO}_{2}<90 \%\right)$ and postoperative nausea and vomiting (PONV) were also recorded.

Statistical analysis. Statistical analysis of the data was performed using SPSS 13.0 (SPSS, Inc., Chicago, IL, USA). Data were presented as the mean \pm standard deviation and analyzed with a Student's t-test while count data were presented as percentage (\%) or median (interquartile range) and analyzed using the $\chi^{2}$ test, respectively. MAP and HR were analyzed using one-way analysis of variance (ANAOVA) followed by Tukey's HSD post hoc test. Grade data, including NRS, OAA/S and VAS, were analyzed using the Mann Whitney-U test. $\mathrm{P}<0.05$ was considered as the level of significant difference. 
Table I. Demographic characteristics of patients.

\begin{tabular}{lccc}
\hline Characteristic & Experimental group (n=31) & Control group (n=31) & P-value \\
\hline Age, years & $35.7 \pm 8.4$ & $36.2 \pm 9.9$ & 0.44 \\
Sex, male/female & $21 / 10$ & $17 / 14$ & 0.43 \\
ASA classification, I/II & $26 / 5$ & $28 / 3$ & 0.71 \\
Height, cm & $169.5 \pm 7.9$ & $166.9 \pm 8.7$ & 0.33 \\
Weight, $\mathrm{kg}$ & $65.3 \pm 10.1$ & $61.9 \pm 9.2$ & 0.67 \\
Body mass index, $\mathrm{kg} / \mathrm{m}^{2}$ & $22.6 \pm 1.8$ & $22.1 \pm 1.4$ & 0.10 \\
\hline
\end{tabular}

Data are presented as the mean \pm standard deviation. American Society of Anesthesiologists.

\section{Results}

Demographic characteristics of patients. A total of 64 patients participated in the present study. Data from 62 patients were successfully collected and analyzed. The patients' characteristics did not differ between the two groups (Table I).

MAP and HR measurement. MAP and HR of the experimental group at T3 and T7 was significantly lower compared with the control group $(\mathrm{P}<0.05$; Fig. 1). There was no significant difference in MAP between the two groups at any other time point. There was no significant difference in HR between the two groups at T0 and T1; however, the HR of the experimental group was significantly lower compared with the control group at T2-T7 (P<0.05; Fig. 2).

Intraoperative variables of patients. Intraoperative observation results are shown in Table II. There was no significant difference between the two groups in terms of the surgical duration or anesthesia duration. In the experimental group, the mean infusion rates of propofol and remifentanil during surgery were $101.5 \pm 8.2 \mu \mathrm{g} \mathrm{kg}^{-1} \mathrm{~min}^{-1}$ and $6.1 \pm 0.2 \mu \mathrm{g} \mathrm{kg}^{-1} \mathrm{~h}^{-1}$ respectively, which was significantly lower than the control group, in which the infusion rates were $117.9 \pm 4.3 \mu \mathrm{g} \mathrm{kg}^{-1} \mathrm{~min}^{-1}$ $(\mathrm{P}=0.001)$ and $9.1 \pm 0.4 \mu \mathrm{g} \mathrm{kg}^{-1} \mathrm{~h}^{-1}(\mathrm{P}=0.045)$, respectively. The incidence rate of bradycardia was higher in the experimental group compared with the control group (22.6 vs. 9.7\%); however, this difference was not statistically significant. Blood loss in the experimental group was $195 \pm 52.5 \mathrm{ml}$, which was significantly lower than the control group $(260.7 \pm 71.6 \mathrm{ml}$; $\mathrm{P}=0.007)$. Surgeon satisfaction scores were significantly higher in the experimental group compared with the control group [median (interquartile range): 8 (7) vs. 7 (6); $\mathrm{P}<0.01$ ].

Recovery outcomes. Postoperative recovery data of the patients were shown in Table III. There was no significant difference in extubation time between the experimental group and the control group $(17.1 \pm 3.2$ vs. $14.8 \pm 2.8 \mathrm{~min} ; \mathrm{P}>0.05)$. There was no significant difference in respiratory rate in PACU between

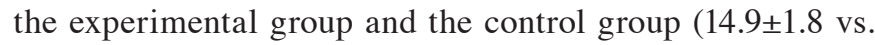
$15.4 \pm 1.9$ beats $\left.\mathrm{min}^{-1}\right)$. The VAS score of the experimental group was significantly improved when compared with the control group [median (interquartile range): 2 (1) vs. 3 (2); $\mathrm{P}<0.01]$. None of the patients were administered fentanyl for analgesic recovery. There was no significant difference in

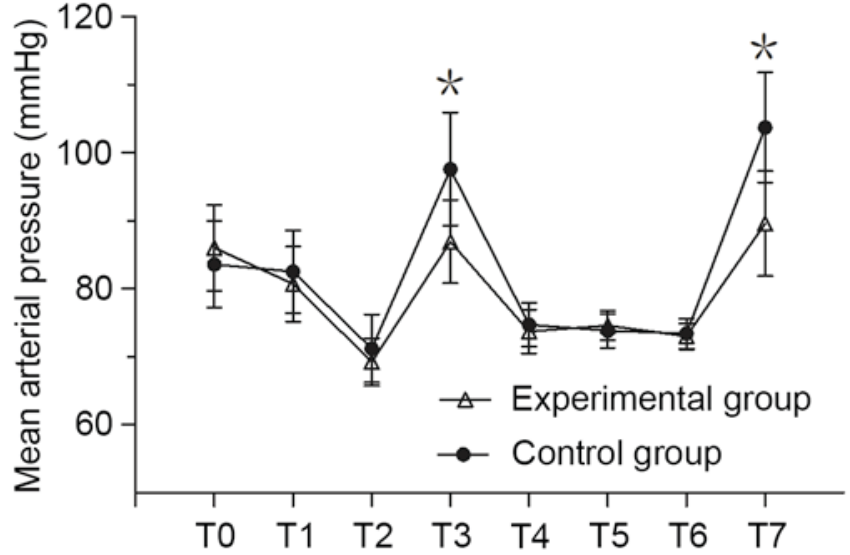

Figure 1. Comparison of mean arterial pressure between the two groups. Data are expressed as the mean \pm standard deviation. ${ }^{*} \mathrm{P}<0.05$ vs. control group. $\mathrm{T} 0$, baseline; $\mathrm{T} 1$, before anesthesia induction; $\mathrm{T} 2$, before tracheal intubation; $\mathrm{T} 3$, at the moment of tracheal intubation; T4, before the start of surgery; T5, 15 min after the start of surgery; T6, at the end of surgery; T7, at the moment of tracheal extubation.

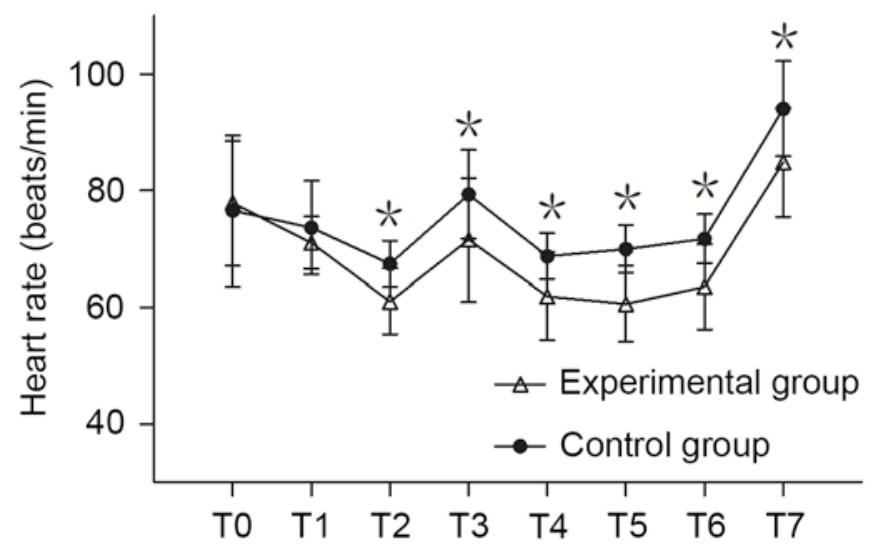

Figure 2. Comparison of heart rate between the two groups. Data are expressed as the mean \pm standard deviation. ${ }^{*} \mathrm{P}<0.05$ vs. control group. T0, baseline; $\mathrm{T} 1$, before anesthesia induction; $\mathrm{T} 2$, before tracheal intubation; $\mathrm{T} 3$, at the moment of tracheal intubation; T4, before the start of surgery; T5, 15 min after the start of surgery; T6, at the end of surgery; T7, at the moment of tracheal extubation.

postoperative OAA/S sedation score between the two groups [median (interquartile range): 1 (1) vs. 1 (1)]. There was no significant difference in the incidence of PONV between the 
Table II. Intraoperative variables.

\begin{tabular}{|c|c|c|c|}
\hline Variable & Experimental group $(n=31)$ & Control group $(n=31)$ & P-value \\
\hline Duration of surgery, min & $70.3 \pm 6.2$ & $83.8 \pm 8.8$ & 0.149 \\
\hline Duration of anesthesia, min & $86.3 \pm 6.0$ & $98.0 \pm 8.8$ & 0.059 \\
\hline Propofol consumption, $\mu \mathrm{g} / \mathrm{kg} / \mathrm{min}$ & $101.5 \pm 8.2$ & $117.9 \pm 4.3$ & 0.001 \\
\hline Remifentanil consumption, $\mu \mathrm{g} / \mathrm{kg} / \mathrm{h}$ & $6.1 \pm 0.2$ & $9.1 \pm 0.4$ & 0.045 \\
\hline Incidence of bradycardia ${ }^{a}$ & $7(22.6)$ & $3(9.7)$ & 0.301 \\
\hline Blood loss, ml & $195.0 \pm 52.5$ & $260.7 \pm 71.6$ & 0.007 \\
\hline Satisfaction score ${ }^{\mathrm{b}}$ & $8(7)$ & $7(6)$ & $<0.01$ \\
\hline
\end{tabular}

Data are presented as the mean \pm standard deviation, ${ }^{a}$ numbers $(\%)$ or ${ }^{b}$ median (interquartile range).

Table III. Recovery profiles of patients.

\begin{tabular}{|c|c|c|c|}
\hline Variable & Experimental group $(n=31)$ & Control group $(n=31)$ & P-value \\
\hline Extubation time, min & $17.1 \pm 3.2$ & $14.8 \pm 2.8$ & 0.422 \\
\hline Respiratory rate in PACU, breaths/min & $14.9 \pm 1.8$ & $15.4 \pm 1.9$ & 0.705 \\
\hline Postoperative pain ${ }^{\mathrm{a}}$ & $2(1)$ & $3(2)$ & $<0.010$ \\
\hline Postoperative sedation $^{\mathrm{a}}$ & $1(1)$ & $1(1)$ & 0.084 \\
\hline Incidence of PONV ${ }^{b}$ & $2(6.5)$ & $4(12.9)$ & 0.671 \\
\hline
\end{tabular}

Extubation time is defined as the time from discontinuation of propofol to extubation. Postoperative pain was assessed via the visual analog scores. Postoperative sedation was assessed via observer's assessment of alertness/sedation scores. Data are presented as the mean \pm standard deviation, ${ }^{a}$ median (interquartile range) or ${ }^{b}$ numbers (\%). PACU, post-anesthetic care unit; PONV postoperative nausea vomiting.

experimental group and control group (6.5 vs. 12.9\%). There was no case of hypoxia in either group.

\section{Discussion}

The results of the present study suggested that target-controlled infusion of propofol and remifentanil combined with dexmedetomidine for FESS was able to reduce the intraoperative bleeding and improve the satisfaction score of the surgical field, compared with target-controlled infusion of propofol and remifentanil alone. Dexmedetomidine also reduced increases in MAP and HR during tracheal intubation and extubation, and reduced postoperative pain.

Surgical bleeding is a critical issue in FESS since bleeding makes the anatomical structure difficult to identify, leading to serious complications and affecting the outcomes of surgery (5). Numerous studies have indicated controlled hypotension with anesthetic or vasoactive drugs may reduce bleeding and improve the quality of FESS (11,14-16). However, vasoactive drugs cannot reduce the FESS bleeding when MAP was controlled at level of 65-75 $\mathrm{mmHg}$ (17); conversely, vascular dilation caused by the drug would increase surgical bleeding and has a negative impact on the surgical field (14). Previous studies have demonstrated a significant correlation between the HR and surgical field bleeding during surgery (18-20). Compared with nitroglycerin, esmolol reduces bleeding by slowing down HR and offering an improved surgical approach with only minimal reduction in MAP (18). Therefore, FESS should be performed at a lower level of HR (20) as controlling HR is more effective on improving the surgical approach than lowering MAP. Compared with inhalation anesthesia, including isoflurane, sevoflurane, total intravenous anesthesia with propofol and remifentanil is able quickly and effectively to lower HR and MAP, reduce bleeding and provide an improved surgical approach $(6-8,21,22)$. The reason for this is that inhalation anesthetics dilate blood vessels and cause reflex tachycardia (23). Although inhalation anesthetics can reduce MAP, the surgical approach was still not improved because of the increased HR and dilated nasal mucosal vessels during FESS (24). Even if balanced anesthesia combined with esmolol controls the HR, the surgical approach is not ameliorated compared with total intravenous anesthesia (25).

Dexmedetomidine is a highly selective $\alpha 2$ adrenoceptor agonist that inhibits norepinephrine release, thereby reducing HR and blood pressure (9). This effect makes it similar to remifentanil $(10,11)$ and esmolol $(26)$ in reducing bleeding and improving the surgical approach. In the present study, total intravenous anesthesia with propofol and remifentanil combined with dexmedetomidine maintained HR at a lower level, reduced bleeding and improved surgical satisfaction scores. In addition, unlike anesthetic drugs which dilate blood vessels, dexmedetomidine constricts peripheral arteries and veins (27). Although there is no clear clinical evidence that dexmedetomidine can contract blood vessels in the nasal mucosa, it is not ruled out that reducing bleeding may be associated with vasoconstriction. 
In order to reduce bleeding, clinical control of hypotension typically controls MAP below the normal range in clinical practice (3). But hypotension reduces visceral blood flow, which causes important organ dysfunction, particularly in elderly patients and patients with organ damage (28). A previous study indicated that it was not necessary to deliberately decrease the MAP to a dangerous level for reaching a clear surgical field (29). In the present study, administration of dexmedetomidine provided good vision during the surgery although MAP was maintained at relatively high level (70-80 mmHg), which increased patient safety.

During surgical procedures, the dosage of anesthetic drugs, including propofol and remifentanil, was increased in order to control blood pressure (10), which resulted in deep anesthesia, thereby affecting the long-term prognosis of patients (30). As an anesthetic adjuvant, dexmedetomidine may reduce the dosage of opioids and propofol (31). In the present study, compared with propofol and remifentanil alone, dexmedetomidine was able to significantly decrease the mean infusion rate of propofol $\left(101.5 \pm 8.2\right.$ vs. $\left.117.9 \pm 4.3 \mu \mathrm{g} \mathrm{kg}^{-1} \mathrm{~min}^{-1}\right)$ and remifentanil $\left(6.1 \pm 0.2\right.$ vs. $\left.9.1 \pm 0.4 \mu \mathrm{g} \mathrm{kg}^{-1} \mathrm{~h}^{-1}\right)$ and reduce the dosage at the same depth of anesthesia. In cases where it is not possible to monitor the depth of anesthesia, this effect may be able to avoid the side effects caused by deep anesthesia.

Dexmedetomidine has also been reported to have additional advantages, including decreasing stress response during tracheal intubation (32) and extubation (33) and improving postoperative analgesia quality (32). In the present study, dexmedetomidine reduced MAP and HR during intubation and extubation and reduced the postoperative VAS value, which is consistent with previous studies $(32,33)$. Guven et al $(34)$ reported that dexmedetomidine was able to reduce the incidence of PONV. This conclusion was not reached in the present study because Guven administered a higher initial dose of dexmedetomidine compared with the present study (1.0 vs. $\left.0.5 \mu \mathrm{g} \mathrm{kg}^{-1} \mathrm{~h}^{-1}\right)$.

However, the present study had some limitations. Firstly, the control group had a relatively high MAP and there was no patient group with low MAP. Therefore, further analysis is required to investigate whether dexmedetomidine can reduce FESS hemorrhage and improve the surgical approach in cases of low MAP. Secondly, the quality of the surgical approach was assessed by the same surgeon in the present study, which may be subjective because different surgeons may give different scores based on proficiency, mental state and other factors. Therefore, large sample, multi-center clinical trials are required to confirm the findings of the present study.

In conclusion, the present study indicated that propofol and remifentanil target-controlled infusion combined with dexmedetomidine for FESS reduced intraoperative bleeding and improved the quality of the surgical field in the case of relatively high MAP. In addition, dexmedetomidine also reduced the increase in MAP and HR during intubation and extubation, and improved the quality of postoperative analgesia.

\section{References}

1. Slack R and Bates G: Functional endoscopic sinus surgery. Am Fam Physician 58: 707-718, 1998.

2. Cinčikas D, Ivaškevičius J, Martinkènas JL and Balseris S: A role of anesthesiologist in reducing surgical bleeding in endoscopic sinus surgery. Medicina (Kaunas) 46: 730-734, 2010.
3. Degoute CS: Controlled hypotension: A guide to drug choice. Drugs 67: 1053-1076, 2007.

4. Ge YL, Lv R, Zhou W, Ma XX, Zhong TD and Duan ML: Brain damage following severe acute normovolemic hemodilution in combination with controlled hypotension in rats. Acta Anaesthesiol Scand 51: 1331-1337, 2007.

5. Amorocho MR and Sordillo A: Anesthesia for functional endoscopic sinus surgery: A review. Anesthesiol Clin 28: 497-504, 2010.

6. Tirelli G, Bigarini S, Russolo M, Lucangelo U and Gullo A: Total intravenous anaesthesia in endoscopic sinus-nasal surgery. Acta Otorhinolaryngol Ital 24: 137-144, 2004.

7. Miłoński J, Zielińska-Bliźniewska H, Golusiński W, Urbaniak J, Sobański R and Olszewski J: Effects of three different types of anaesthesia on perioperative bleeding control in functional endoscopic sinus surgery. Eur Arch Otorhinolaryngol 270: 2045-2050, 2013.

8. Marzban S, Haddadi S, Mahmoudi Nia H, Heidarzadeh A, Nemati S and Naderi Nabi B: Comparison of surgical conditions during propofol or isoflurane anesthesia for endoscopic sinus surgery. Anesth Pain Med 3: 234-238, 2013.

9. Gerlach AT and Dasta JF: Dexmedetomidine: An updated review. Ann Pharmacother 41: 245-252, 2007.

10. Kim H, Ha SH, Kim CH, Lee SH and Choi SH: Efficacy of intraoperative dexmedetomidine infusion on visualization of the surgical field in endoscopic sinus surgery. Korean J Anesthesiol 68: 449-454, 2015.

11. Lee J, Kim Y, Park C, Jeon Y, Kim D, Joo J and Kang H: Comparison between dexmedetomidine and remifentanil for controlled hypotension and recovery in endoscopic sinus surgery. Ann Otol Rhinol Laryngol 122: 421-426, 2013.

12. Yeganeh N, Roshani B, Latifi H and Almasi A: Comparison of target-controlled infusion of sufentanil and remifentanil in blunting hemodynamic response to tracheal intubation. J Inj Violence Res 5: 101-107, 2013.

13. Hall DL, Weaver J, Ganzberg S, Rashid R and Wilson S: Bispectral EEG index monitoring of high-dose nitrous oxide and low-dose sevoflurane sedation. Anesth Prog 49: 56-62, 2002.

14. Boezaart AP, van der Merwe J and Coetzee A: Comparison of sodium nitroprusside- and esmolol-induced controlled hypotension for functional endoscopic sinus surgery. Can J Anaesth 42: 373-376, 1995.

15. Crawley BK, Barkdull GC, Dent S, Bishop M and Davidson TM: Relative hypotension and image guidance: Tools for training in sinus surgery. Arch Otolaryngol Head Neck Surg 135: 994-999, 2009.

16. Cardesín A, Pontes C, Rosell R, Escamilla Y, Marco J, Escobar MJ and Bernal-Sprekelsen M: Hypotensive anaesthesia and bleeding during endoscopic sinus surgery: An observational study. Eur Arch Otorhinolaryngol 271: 1505-1511, 2014.

17. Jacobi KE, Böhm BE, Rickauer AJ, JacobiC and Hemmerling TM: Moderate controlled hypotension with sodium nitroprusside does not improve surgical conditions or decrease blood loss in endoscopic sinus surgery. J Clin Anesth 12: 202-207, 2000.

18. Srivastava U, Dupargude AB, Kumar D, Joshi K and Gupta A: Controlled hypotension for functional endoscopic sinus surgery: Comparison of esmolol and nitroglycerine. Indian J Otolaryngol Head Neck Surg 65: 440-444, 2013.

19. Wormald PJ, Athanasiadis T, Rees G and Robinson S: An evaluation of effect of pterygopalatine fossa injection with local anesthetic and adrenalin in the control of nasal bleeding during endoscopic sinus surgery. Am J Rhinol 19: 288-292, 2005.

20. Nair S, Collins M, Hung P, Rees G, Close D and Wormald PJ: The effect of beta-blocker premedication on the surgical field during endoscopic sinus surgery. Laryngoscope 114: 1042-1046, 2004.

21. Eberhart LH, Folz BJ, Wulf H and Geldner G: Intravenous anesthesia provides optimal surgical conditions during microscopic and endoscopic sinus surgery. Laryngoscope 113: 1369-1373, 2003.

22. Wormald PJ, van Renen G,Perks J, Jones JA and Langton-Hewer CD: The effect of the total intravenous anesthesia compared with inhalational anesthesia on the surgical field during endoscopic sinus surgery. Am J Rhinol 19: 514-520, 2005.

23. Toivonen J, Virtanen H and Kaukinen S: Deliberate hypotension induced by labetalol with halothane, enflurane or isoflurane for middle-ear surgery. Acta Anaesthesiol Scand 33: 283-289, 1989.

24. Snidvongs K, Tingthanathikul W, Aeumjaturapat S and Chusakul S: Dexmedetomidine improves the quality of the operative field for functional endoscopic sinus surgery: Systematic review. J Laryngol Otol 129: S8-13, 2015. 
25. Ragab SM and Hassanin MZ: Optimizing the surgical field in pediatric functional endoscopic sinus surgery: A new evidence-based approach. Otolaryngol Head Neck Surg 142: 48-54, 2010.

26. Shams T, El Bahnasawe NS, Abu-Samra M and El-Masry R: Induced hypotension for functional endoscopic sinus surgery: A comparative study of dexmedetomidine versus esmolol. Saudi J Anaesth 7: 175-180, 2013.

27. Talke P, Lobo E and Brown R: Systemically administered alpha2-agonist-induced peripheral vasoconstriction in humans. Anesthesiology 99: 65-70, 2003.

28. Larsen R and Kleinschmidt S: Controlled hypotension. Anaesthesist 44: 291-308, 1995.

29. Sieśkiewicz A, Drozdowski A and Rogowski M: The assessment of correlation between mean arterial pressure and intraoperative bleeding during endoscopic sinus surgery in patients with low heart rate. Otolaryngol Pol 64: 225-228, 2010.
30. Monk TG, Saini V, Weldon BC and Sigl JC: Anesthetic management and one-year mortality after noncardiac surgery. Anesth Analg 100: 4-10, 2005.

31. Arcangeli A, D'Alò C and Gaspari R: Dexmedetomidine use in general anaesthesia. Curr Drug Targets 10: 687-695, 2009.

32. Gunalan S, Venkatraman R, Sivarajan G and Sunder P: Comparative evaluation of bolus administration of dexmedetomidine and fentanyl for stress attenuation during laryngoscopy and endotracheal intubation. J Clin Diagn Res 9: UC06-UC09, 2015.

33. Fan Q, Hu C, Ye M and Shen X: Dexmedetomidine for tracheal extubation in deeply anesthetized adult patients after otologic surgery: A comparison with remifentanil. BMC Anesthesiol 15: $106,2015$.

34. Guven DG, Demiraran Y, Sezen G, Kepek O and Iskender A: Evaluation of outcomes in patients given dexmedetomidine in functional endoscopic sinus surgery. Ann Otol Rhinol Laryngol 120: 586-592, 2011. 